Background/introduction Missed opportunities is a leading cause for late presentation in HIV.

Aim(s)/objectives We analysed missed opportunities, clinical outcomes and associated cost in a HIV low prevalence region in UK.

Methods A retrospective review of case notes and pathology system of new HIV diagnosis from 2010 to 2013 was undertaken. Clinical summary preceding 12 months of diagnosis collected from GPs with patient's consent. Data analysed using Excel workbook.

Results Out of 25 new HIV diagnosis, 17 males, 21 white ethnicity, 10 heterosexual and 6 bisexual. One third $>40$ years. Sixteen $(64 \%)$ were late diagnosis with CD4 < 350. 13/16 had CD4 < 200 and 9/16 (56\%) had an AIDS defining illness. 19/25 (76\%) had atleast one missed opportunity (range 1-16). 11/19 in primary care and remaining at different levels. There was no difference in VL between early and late diagnosis. 10/16 had a blood test in the preceding 12 months. In the first 12 months post diagnosis, early group had 51 clinical consultation compared to 147 in late group. Three patients had extended inpatient stay in the late group.One died. Using Reference costs of around $£ 385$, late diagnosis costed $£ 56595$ compared to $£ 19635$ for early excluding inpatients cost, excess bed days, additional outpatient investigations, medications including ARVS and other specialty referral costs.

Discussion/conclusion Our study shows increased missed opportunities in apparently non high-risk groups resulting in poor outcomes and significant costs. Higher HIV awareness and national testing policy tailored to HIV low prevalence region is required.

\section{P225 LYMPHOGRANULOMA VENEREUM: CASE REPORT OF SIMULTANEOUS PENILE ULCERATION AND BUBO FORMATION}

Elizabeth Okecha*, Ashish Sukthankar. Central Manchester University Hospitals NHS Foundation Trust, Manchester, UK

\subsection{6/sextrans-2016-052718.273}

Background/introduction Lymphogranuloma venereum (LGV) has become an important UK public health issue since 2003. HIV positive men who have sex with men (MSM) continue to be the predominant risk group for contracting LGV in the UK.

Aim(s)/objectives LGV commonly presents as proctitis alone; we present an unusual case of a patient who presented with simultaneous rectal symptoms, penile ulceration and bubo.

Methods A 25 year old HIV (CD4 996 (43\%)/viral load undetectable) and Hepatitis C co-infected MSM presented to A\&E with a 2 week history of painful left inguinal swelling. Additional history revealed episodes of bloody diarrhoea which the patient related to a previous diagnosis of Inflammatory Bowel disease. Examination revealed a left inguinal bubo and 3 areas of penile ulceration (2 painful, one indurated/painless). Proctoscopy was normal. Empirical treatment with Doxycycline and Aciclovir was commenced.

Results Nucleic acid amplification testing (NAAT) was performed on pharynx, rectum, urine and penile ulcers. Urine NAAT was positive for Chlamydia trachomatis; penile ulcer NAAT was positive for LGV specific DNA. Herpes simplex and Treponemal PCR from the ulcers were negative. Rectal NAAT was negative for Chlamydia trachomatis. An Ultrasound of the left inguinal bubo revealed abscess formation with frank pus seen on aspiration.
Discussion/conclusion LGV is commonly associated with proctitis and genital ulcers of LGV are evanescent. Persistence of LGV ulceration until bubo formation has not been reported to-date. The negative Rectal NAAT for Chlamydia was unusual however the diarrhoeal symptoms settled with Doxycycline.

\section{P226 REATTENDANCE, RETESTING AND TEST POSITIVITY AMONG PATIENTS INITIALLY TREATED FOR CHLAMYDIA OR GONORRHOEA IN A LARGE INNER CITY CLINIC}

Sarah Bradley*, John Saunders, Eva Jungmann. Mortimer Market Centre, CNWL, London, UK

\subsection{6/sextrans-2016-052718.274}

Background/introduction Reinfection with a bacterial sexually transmitted infection following treatment is common. National guidelines recommend retesting for chlamydia three to six months after treatment in $<25$ year olds.

Aim(s)/objectives To review reattendance, retest and positivity rates in patients with chlamydia or gonorrhoea at a large innercity clinic.

Methods Patients diagnosed with chlamydia or gonorrhoea were identified in our electronic patient records (March-September 2015). Proportion reattending, retesting and test positivity two to four months after treatment of the initial infection were recorded.

Results The minority of patients treated for chlamydia and gonorrhoea reattended and retested for infection (Table 1). Positivity was high in those who retested, especially in MSM. Two HIV diagnoses and 89 syphilis serology positive results were found in those retesting.

Abstract P226 Table 1 Reattendance, retesting and test positivity among patients initially treated for chlamydia or gonorrhoea, by age and risk group

\begin{tabular}{lllll}
\hline & $<25$ years $(n=584)$ & $\geq 25$ years $(n=1,713)$ \\
\hline & $\begin{array}{l}\text { Heterosexual } \\
(n=442)\end{array}$ & $\begin{array}{l}\text { MSM } \\
(n=142)\end{array}$ & $\begin{array}{l}\text { Heterosexual } \\
(n=647)\end{array}$ & $\begin{array}{l}\text { MSM } \\
(n=1066)\end{array}$ \\
\hline $\begin{array}{l}\text { Total: Chlamydia } \pm \\
\text { Gonorrhoea }(\mathrm{n}=2297)\end{array}$ & & & & \\
Reattended & $97(22 \%)$ & $42(30 \%)$ & $148(23 \%)$ & $481(45 \%)$ \\
$\begin{array}{l}\text { Retested } \\
\text { Positivity }\end{array}$ & $76(78 \%)$ & $25(60 \%)$ & $96(65 \%)$ & $258(54 \%)$ \\
\hline & $11(14 \%)$ & $9(36 \%)$ & $11(11 \%)$ & $92(36 \%)$ \\
\hline
\end{tabular}

Discussion/conclusion Retesting rates following treatment for chlamydia and gonorrhoea are low. Those who did reattend and retest experienced high rates of infections and represent an important group to target for active recall.

\section{P227 PATIENT SATISFACTION - NEW LGBTQ (LESBIAN, GAY, BISEXUAL AND QUEER) SEXUAL HEALTH CLINIC}

Grainne Cooney*, Eleanor Hamlyn, Sabrina Wallace, Ella Svensson, Michael Underwood, Tristan Barber. John Hunter Clinic, Chelsea and Westminster Hospital., London, UK

\subsection{6/sextrans-2016-052718.275}

Background/introduction This clinic opened in 2015 offering a weekly specialist sexual health service for our LGBTQ community. Burden of pathology is high, suggesting a potentially more 
stressful environment for patients. All medical and non-medical staff were trained on LGBTQ sexual health issues and some on Club Drug related issues.

Aim To assess patient satisfaction with the new service, a questionnaire was undertaken seven months after service commencement.

Methods Over a four-week period, questionnaires were handed to patients by reception at booking. Thirty-six questionnaires were returned.

Results Patients rated the service as excellent $81 \%$ (26/32) very good 14\% (5/32) or good 3\% (1/32). All patients who answered said they would attend again $100 \%(32 / 32)$. Most would recommend it to a friend $97 \%(32 / 33)$. Patient staff satisfaction was high, with 96\% (29/30) stating they felt listened to. Patients felt treated with respect and dignity always 97\% (31/32) or sometimes $3 \%(1 / 32) .83 \%(30 / 36)$ of patients attended specifically with LGBTQ concerns, of these 93\% (28/30) felt confident in discussing concerns with staff, $7 \%(2 / 30)$ to some extent. $77 \%$ (24/31) indicated that drugs or alcohol were related to their visit. Only $21 \%(5 / 24)$ felt they could raise this topic with staff and $79 \%(19 / 24)$ stated they couldn't.

Discussion Staff training on managing vulnerable clients' health needs enables confidant and approachable health care. This provides patients with opportunities to verbalise health anxieties, facilitating opportunistic healthcare interventions. Drug and Alcohol training for all staff will enhance the patient care package.

\section{P228 EVALUATING THE USE OF EXTRA-GENITAL CHLAMYDIA TRACHOMATIS (CT) AND NEISSERIA GONORRHOEA (GC) NUCLEIC ACID AMPLIFICATION TESTS (NAATS) IN NON- MSM POPULATIONS}

Maria Elbadawi*, Rosemary Lockyer, Amy Bennett. Ashford and St Peter's Hospitals NHS Foundation Trust, Chertsey, UK

\subsection{6/sextrans-2016-052718.276}

Background/introduction Our clinic performs routine oral and anal CT and GC NAATs following disclosure during sexual history of oral or receptive anal sex. Specimens are analysed using the Abbott M2000 assay, costing $£ 4.50$ per dual NAAT.

$\operatorname{Aim}(\mathrm{s}) /$ objectives We report the findings of this practice, focusing on clinical and partner notification outcomes, and the cost per diagnosis.

Methods We included all non-MSM patients with isolated extragenital CT or GC infection from $1 / 8 / 14$ to $31 / 7 / 15$. We excluded contacts of either infection, plus those without GC confirmed by culture or second NAAT platform. Retrospective case note review was performed for identified cases.

Results In total 8796 NAATs were processed in non-MSM patients, of which 3813 (43\%) were extra-genital. In heterosexual men we identified 3 pharyngeal GC cases and one contact, and one pharyngeal CT case with one contact. In women there were 2 pharyngeal GC cases, one pharyngeal CT case and one rectal CT case with no additional contacts. The spend per pharyngeal diagnosis was $£ 1519$ in males and $£ 3138$ in women. The spend per rectal diagnosis in women was $£ 1665$.

Discussion/conclusion Our screening practice has picked up only a small number of cases which would otherwise have been missed. $25 \%(2 / 8)$ of those cases had verified contacts identified. The cost implication of such screening is high, meaning that ongoing routine screening is not viable. In our relatively low- prevalence setting we now intend to target our screening in line with BASHH CEG guidance which will provide significant cost savings.

\section{P229 CLINICIAN AND COMMUNITY COLLABORATION ON PREP IN THE UK - A NARRATIVE}

${ }^{1}$ Mags Portman*, ${ }^{2}$ GrEG Owen, ${ }^{3}$ Killian Quinn, ${ }^{2}$ Alex Craddock, ${ }^{8}$ Marc Thompson, ${ }^{4}$ Nneka Nwokolo, ${ }^{5}$ lain Reeves, ${ }^{6}$ Jake Bayley, ${ }^{7}$ Michael Brady, ${ }^{8}$ Will Nutland, ${ }^{4,9}$ Sheena McCormack. 'Mortimer Market Centre, Central and North West London NHS Foundation Trust, London, UK; ${ }^{2}$ WantPrEPNow.co.uk, London, UKi ${ }^{3}$ King's College Hospital NHS Foundation Trust, London, UKi ${ }^{4} 56$ Dean Street, Chelsea and Westminster Hospital NHS Foundation Trust, London, UK; ${ }^{5}$ Homerton University Hospital NHS Foundation Trust, London, UK; ${ }^{6}$ Barts Health NHS Trust, London, UK; ${ }^{7}$ Terence Higgins Trust, London, UK; ${ }^{8}$ Prepster.info, London, UK; ${ }^{9}$ Medical Research Unit Clinical Trials Unit at UCL London, UK

\subsection{6/sextrans-2016-052718.277}

Background The PROUD study showed that PrEP can be successfully provided via sexual health clinics in the UK. The delay in widespread availability of PrEP has seen renewed grassroots activism from the community sector.

Methods We document the timeline and benefits of a close relationship between community activists and clinicians in advancing the PrEP agenda.

Results Community engagement during the PROUD trial led on to increased advocacy and activism from October 2014 when interim results were published. Launch of the websites IWantPrEPNow.co.uk (with a click to buy generic PrEP) and PrEPster. info in October 2015 further galvanised clinicians and community advocates into greater joined up action. The benefits of this included: 1) Mutual social media support; advancing the PrEP agenda amongst potential PrEP users and clinicians, 2) Obtaining GMC input on providing clinical advice regarding generics, 3) Pushing for free monitoring for current generic PrEP users, 4) Sharing of therapeutic drug monitoring data, 5) Community input into staff training and service development such as production and review of patient information, and 6) Two way learning; sharing PrEP users perspective and evidence based research. Discussion/conclusion The lack of availability of PrEP in the UK is proving a challenge to both community advocates and clinicians. We wish to celebrate the achievements made within the confines of the current political climate, in particular working to ensure patient safety. There is a history of successful clinician/ community collaboration in GUM/HIV medicine; with an NHS England PrEP roll out we need to reflect this to drive uptake. If NHS England decides against PrEP funding, we suggest that continued collaboration, support and innovation will be paramount.

\section{P230 REVIEW OF SYPHILIS MANAGEMENT AT A BUSY URBAN STI CLINIC}

Niamh Lynn*, Padraig Calpin, Brendan O'Kelly, Ksenia Davenport, Fiona Lyons. GUIDe Clinic, St. James's Hospital, Dublin, Ireland

\subsection{6/sextrans-2016-052718.278}

Background/introduction Appropriate management of syphilis is vital in order to avoid late complications. A retrospective cohort study of patients with positive serology at an urban STI clinic where the BASHH guidelines are followed was undertaken. 